\title{
Comparison of the Enhancement of Plasma Glucose Levels in Type 2 Diabetes Otsuka Long-Evans Tokushima Fatty Rats by Oral Administration of Sucrose or Maple Syrup
}

\author{
Noriaki Nagai, Yoshimasa Ito and Atsushi Taga* \\ Faculty of Pharmacy, Kinki University, 3-4-1 Kowakae, Higashi-Osaka, Osaka, 577-8502, Japan.
}

\begin{abstract}
Maple syrup is used as a premium natural sweeter, and is known for being good for human health. In the present study, we investigate whether maple syrup is suitable as a sweetener in the management of type 2 diabetes using Otsuka Long-Evans Tokushima Fatty (OLETF) rats, a model of type 2 diabetes mellitus. OLETF rats develop type 2 diabetes mellitus by 30 weeks of age, and 60-week-old OLETF rats show hyperglycemia and hypoinsulinemia via pancreatic $\beta$-cell dysfunction. The administration of sucrose or maple syrup following an OGT test increased plasma glucose (PG) levels in OLETF rats, but the enhancement in PG following the oral administration of maple syrup was lower than in the case of sucrose administration in both 30- and 60-week-old OLETF rats. Although, the insulin levels in 30-week-old OLETF rats also increased following the oral administration of sucrose or maple syrup, no increase in insulin levels was seen in 60-week-old OLETF rats following the oral administration of either sucrose or maple syrup. No significant differences were observed in insulin levels between sucrose- and maple syrup-administered OLETF rats at either 30 or 60 weeks of age. The present study strongly suggests that the maple syrup may have a lower glycemic index than sucrose, which may help in the prevention of type 2 diabetes.
\end{abstract}

Key words: Maple syrup, plasma glucose, type 2 diabetes mellitus, insulin, OGT test, Otsuka Long-Evans Tokushima Fatty rat

\section{INTRODUCTION}

Persons with metabolic syndrome are at increased risk for developing type 2 diabetes mellitus and cardiovascular disease as well as for increased mortality from cardiovascular diseases and all other causes ${ }^{1,2}$. The prevalence of type 2 diabetes mellitus resulting from metabolic syndrome is increasing rapidly, and is affecting the health of millions of humans, and will continue to do so in the near future. The development of type 2 diabetes mellitus is associated with pancreatic $\beta$-cell dysfunction occurring together with insulin resistance(IR). Normal $\beta$-cells can compensate for IR by increasing insulin secretion, but insufficient compensation leads to the onset of glucose intolerance. Once hyperglycemia becomes apparent, $\beta$-cell function progressively deteriorates: glucose-induced insulin secretion becomes further impaired and degranulation of $\beta$-cells becomes evident, often accompanied by a decrease in the number of $\beta$-cells ${ }^{3-6)}$. Recently, it was reported that the production of reactive oxygen species (ROS) mediated by glycation reduces insulin gene transcription and decreases the number of $\beta$-cells through apoptosis ${ }^{7,8)}$. Once $\beta$-cells are exposed to ROS under diabetic conditions, they are likely to be profoundly damaged due to their low levels of antioxidant enzyme expression ${ }^{9)}$. Therefore, the identification of sweeteners with lower glycemic indices and antioxidant activity is highly expected as a way to improve the quality of life for patients with type 2 diabetes mellitus.

Maple syrup is a natural sweeter consumed by many people of all ages throughout the world. Apart from sucrose, which is its dominant sugar, maple syrup contains organic acids, amino acids, vitamins and minerals including manganese and zinc $^{10-14)}$. Recent studies have shown that maple syrup contains phenolic compounds such as lignans, as well as coumarins ${ }^{15,16)}$ and quebecol ${ }^{17)}$. In addition, the phenolic compounds in maple syrup may possess antioxidative activity ${ }^{18)}$, and an in vitro study of a butanol extract

\footnotetext{
*Correspondence to: Atsushi Taga, Faculty of Pharmacy, Kinki University, 3-4-1 Kowakae, Higashi-Osaka, Osaka, 577-8502, Japan. E-mail: punk@phar.kindai.ac.jp Accepted March 29, 2013 (received for review February 14, 2013) Journal of Oleo Science ISSN 1345-8957 print / ISSN 1347-3352 online http://www.jstage.jst.go.jp/browse/jos/ http://mc.manusriptcentral.com/jjocs
} 
of maple syrup demonstrated an inhibitory activity toward $\alpha$-glucosidase ${ }^{19)}$. Although, these data suggest the possibility that maple syrup might be used to prevent obesity and the development of diabetes mellitus in comparison with other sweeteners such as sucrose and glucose-fructose syrup, there are almost no reports or scientific evidence to indicate the effects of the oral consumption of maple syrup on plasma glucose $(\mathrm{PG})$ levels. In this study, we investigate whether maple syrup is suitable for use as a sweetener in the management of type 2 diabetes mellitus using the Otsuka Long-Evans Tokushima Fatty (OLETF) rats, a model of type 2 diabetes mellitus ${ }^{20)}$.

\section{EXPERIMENTAL}

\subsection{Animals and materials}

Male Long-Evans Tokushima Otsuka rats (LETO, normal control rat) and OLETF rats aged 30 and 60 weeks were used in this study. The LETO and OLETF rats were obtained from Otsuka Pharmaceutical Co., Ltd., and housed under standard conditions $(12 \mathrm{~h} / \mathrm{d}$ fluorescent light (07:00$19: 00), 25^{\circ} \mathrm{C}$ room temperature) with free access to a commercial diet (CE-2, Clea Japan Inc., Tokyo, Japan) and water. All procedures were performed in accordance with the guidelines of the Kinki University Faculty of Pharmacy Committee for the Care and Use of Laboratory Animals. Maple sugar was a gift from BASCOM MAPLE FARMS, INC. (Quebec, Canada), and the carbohydrate composition was analyzed by the Japan Inspection Association of Food and Food Industry Environment by anion exchange HPLC (sucrose; $93.7 \%$, glucose; $0.6 \%$, fructose; not detectable (N.D.), maltose; N.D., galactose; N.D.). The maple syrup used in this study was prepared by freshly desolving maple sugar in water at a common concentration before use to control quality since there was the possibility that a stock of maple syrup could be denatured by bacteria over the course of this long term study (ca. 8 months). The maple sugar concentration was adjusted to $0.8 \mathrm{~g} / \mathrm{ml}$, which was chosen to yield a sugar composition equal to that of the sucrose solution used $(0.75 \mathrm{~g} / \mathrm{ml})$. The resulting the maple syrup contains a higher glucose unit than sucrose because maple sugar contains glucose as a monosaccharide. All other chemicals used were of the highest grade commercially available.

\subsection{Measurement of body weight and blood tests for dia- betes mellitus}

Body weights and some blood test parameters for diabetes mellitus were measured for rats 30 and 60 weeks of age. Blood was drawn from the tail vein of each rat fasted for $15 \mathrm{~h}$ without anesthesia, and plasma glucose (PG), triglycerides (TG), total cholesterol (Total-cho) and insulin levels were measured. The PG and TG levels were deter- mined by the Accutrend GCT (Roche Diagnostics, Mannheim, Germany). Total-cho levels were measured by the cholesterol oxidase method and phosphotungstatemagnesium salt method using a Cholesterol E-Test Kit (Wako, Osaka, Japan). Insulin levels were measured using an ELISA Insulin Kit according to the manufacturer's instructions (Morinaga Institute of Biological Science Inc., Kanagawa, Japan). Briefly, monoclonal antibodies specific for rat insulin were pre-coated onto microplates, standards and samples were pipetted into the wells, and the microplates were incubated at $4^{\circ} \mathrm{C}$ for $2 \mathrm{~h}$. After washing to remove unbound materials, rat insulin antibodies were added to the wells at room temperature for $30 \mathrm{~min}$. After washing, the substrates were added. The enzyme reactions yielded blue products that turned yellow when the stop solutions were added. Insulin levels were determined by measuring the absorbance at $450 \mathrm{~nm}$ with a microplate reader (BIO-RAD, California, USA).

\subsection{Oral glucose tolerance test (OGT test) using sucrose and maple syrup}

Rats were fasted for $15 \mathrm{~h}$, and sucrose or maple syrup (an amount equivalent to $1.5 \mathrm{~g} / \mathrm{kg}$ sucrose) was administered orally to each rat. Blood samples were taken from the tail veins at 0 (just before glucose administration), 15, 30, 60, 120 and $180 \mathrm{~min}$. PG and insulin levels were determined by the Accutrend GCT and ELISA Insulin Kit, respectively.

The differences in the $\mathrm{PG}\left(\Delta C_{\mathrm{PG}}, \mathrm{mg} / \mathrm{dl}\right)$ and insulin $\left(\Delta C_{\text {insulin }}, \mathrm{ng} / \mathrm{dl}\right)$ concentrations between OLETF rats with or without the oral administration of sucrose or maple syrup were analyzed, and the area under the curve for $\Delta C_{\mathrm{PG} \text { or insulin }}\left(A U C_{0-180 \text { min }}\right)$ was calculated according to the following equation:

$$
A U C_{0-180 \min }=\int_{0 \min }^{180 \min } \quad \Delta C_{\mathrm{PG} \text { or insulin }} d t
$$

Briefly, $t$ is time (min)after the oral administration of sucrose or maple syrup. $A U C_{0-180 \text { min }}$ was determined according to the trapezoidal rule up to $180 \mathrm{~min}$, which was the last point of PG or insulin concentration measurement.

\subsection{Statistical analysis}

All data are expressed as the mean \pm standard error (S.E.) of the mean. Unpaired Student's or Aspin-Welch's $t$-test was used to evaluate statistical difference. $P$ values less than 0.05 were considered significant.

\section{RESULTS}

3.1 Changes in plasma glucose levels in 30- or 60-weekold OLETF rats following the oral administration of sucrose or maple syrup

Table 1 shows the changes in food and water intake of LETO and OLETF rats at 30 and 60 weeks of age. The food 
and water intakes of OLETF rats were significantly higher than those of LETO rats, with 60-week-old OLETF rats consuming $40.2 \pm 1.9 \mathrm{~g} /$ day/rat and drinking $81.8 \pm 3.4 \mathrm{~mL} /$ day/rat. The food and water intakes of OLETF rats at 30 and 60 weeks of age were similar in this study. Table 2 shows the body weights, PG, TG, Total-cho and insulin levels of LETO and OLETF rats at 30 and 60 weeks of age. Type 2 diabetes mellitus had developed in the 30 -week-old OLETF rats with metabolic syndrome, and the body weights, PG, TG, Total-cho and insulin levels were all significantly higher than in LETO rats. With continued aging, the 60-week-old OLETF rats had developed hypoinsulinemia, and the body weights and plasma insulin levels were lower than those of LETO rats. Figure 1 shows the influence of the type of sweetener, sucrose and maple syrup, on PG levels. The values of the $A U C_{0-180 \text { min }}$ for $\mathrm{PG}$ in 30- and 60-week-old LETO and OLETF rats administered sucrose or maple syrup are summarized in Table 3. The PG levels of 30- and 60-week-old LETO rats reached a peak 30 - 60 min after the oral administration of sucrose or maple syrup, and then gradually decreased. Consistent with the results in LETO rats, the PG levels in OLETF rats at 30 and 60 weeks of age did not decrease to the pre-prandial levels by $3 \mathrm{~h}$ after the oral administration of either sucrose or maple syrup. The enhancement in PG in 30- and 60-weekold OLETF rats administered maple syrup was lower than that in OLETF rats administered sucrose, and the $A U C_{0-180}$ min values for PG in 30- and 60-week-old OLETF rats administered maple syrup were significantly lower than those in sucrose-administered OLETF rats (ca. 30\% lower, Table 3).

\subsection{Changes in insulin levels in 30- and 60-week-old OLETF rats following the oral administration of su- crose or maple syrup}

Figure 2 shows the influence of the sweetener, sucrose or maple syrup, on plasma insulin levels in the OGT test, and Table 4 shows the $A U C_{0-180 \text { min }}$ for insulin in 30- and 60-week-old LETO and OLETF rats administered sucrose or maple syrup. The insulin levels of 30 and 60 -week old LETO rats reached a peak at 60 min after the oral administration of either sucrose or maple syrup, and then gradually decreased. The insulin levels in OLETF rats at 30 weeks of age were also increased by the oral administration of sucrose or maple syrup, although the enhancement was lower than that in LETO rats, and the insulin levels did not decrease to the pre-prandial levels by $3 \mathrm{~h}$ after the oral administration. On the other hand, the insulin levels in OLETF rats at 60 weeks of age did not increased following the oral administration of either sucrose or maple syrup. No significant differences were observed in the plasma insulin levels between sucrose- and maple syrup-administered LETO or OLETF rats (Table 4).

\section{DISCUSSION}

The restriction of sweeteners, such as sucrose, maple syrup, honey etc., is important to prevent obesity and the promotion of diabetes mellitus. Recently, the results of in vitro studies have suggested that the natural sweetener maple syrup may have antioxidative activity ${ }^{18)}$ and $\alpha$-glucosidase inhibitory activity ${ }^{19)}$. Therefore, it is possible that maple syrup is suitable for use as a natural sweetener

Table 1 Food and water intake of LETO and OLETF rats in this study.

\begin{tabular}{cccccc}
\hline & \multicolumn{2}{c}{ Food intake $(\mathrm{g} /$ day/rat $)$} & & \multicolumn{2}{c}{ Water intake $(\mathrm{ml} /$ day/rat $)$} \\
\cline { 2 - 3 } \cline { 5 - 6 } & 30 weeks & 60 weeks & & 30 weeks & 60 weeks \\
\hline LETO & $24.6 \pm 1.1$ & $28.5 \pm 2.1$ & & $38.4 \pm 1.2$ & $44.0 \pm 1.7$ \\
OLETF & $37.4 \pm 1.8^{*}$ & $40.2 \pm 1.9^{*}$ & & $80.7 \pm 3.3^{*}$ & $81.8 \pm 3.4^{*}$ \\
\hline
\end{tabular}

The data are presented as means \pm S.E. of 6 independent rats. $* p<0.05$ vs. LETO rats for each category.

Table 2 Blood test values for diabetes mellitus in LETO and OLETF rats.

\begin{tabular}{lccccc}
\hline & \multicolumn{2}{c}{30 weeks } & & \multicolumn{2}{c}{60 weeks } \\
\cline { 2 - 3 } \cline { 5 - 6 } & LETO & OLETF & & LETO & OLETF \\
\hline Body weight $(\mathrm{g})$ & $493.3 \pm 8.8$ & $653.3 \pm 5.3^{*}$ & & $483.8 \pm 8.1$ & $397.9 \pm 8.8^{*}$ \\
PG (mg/dl) & $122.5 \pm 1.9$ & $222.3 \pm 5.6^{*}$ & & $124.0 \pm 4.6$ & $274.4 \pm 5.3^{*}$ \\
TG (mg/dl) & $122.4 \pm 9.7$ & $380.9 \pm 14.5^{*}$ & & $129.1 \pm 11.4$ & $353.5 \pm 13.6^{*}$ \\
Total-cho (mg/dl) & $101.6 \pm 17.1$ & $177.1 \pm 15.3^{*}$ & & $103.6 \pm 13.1$ & $267.0 \pm 14.9^{*}$ \\
Insulin (ng/dl) & $109.4 \pm 4.1$ & $313.4 \pm 15.5^{*}$ & & $109.4 \pm 4.7$ & $60.0 \pm 2.1^{*}$ \\
\hline
\end{tabular}

The data are presented as means \pm S.E. of 6 independent rats. $* p<0.05$ vs. LETO rats for each category. 

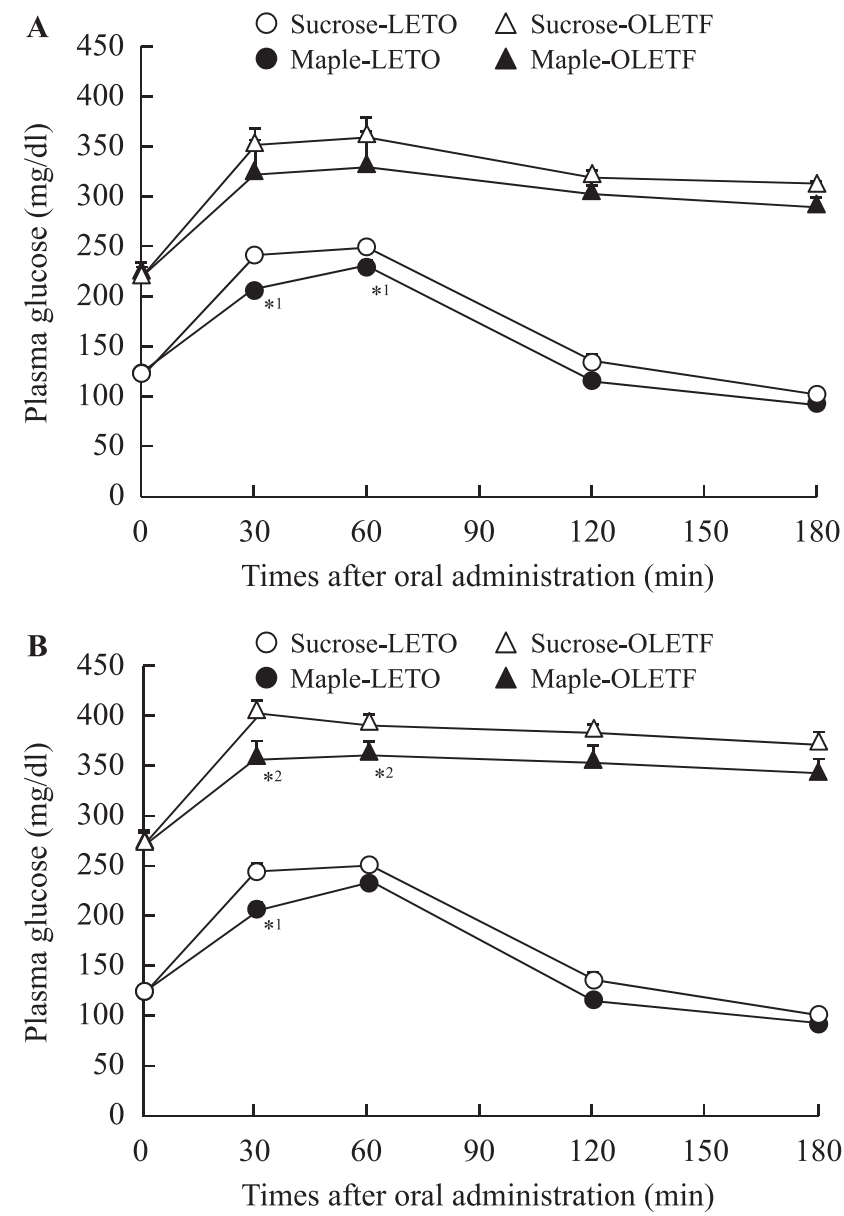

Fig. 1 Changes in PG levels in 30 (A)- and 60 (B)week-old LETO and OLETF rats following the oral administration of sucrose or maple syrup. The PG level was determined by the accutrend GCT. Open circles, sucrose-administered LETO rats; closed circles, maple syrup-administered LETO rats; open triangles, sucrose-administered OLETF rats; closed triangles, maple syrup-administered OLETF rats. The data are presented as means \pm S.E. of 3 independent rats. ${ }^{* 1} p<0.05$ vs. sucrose-administered LETO rats for each category. ${ }^{2} p<0.05$ vs. sucrose-administered OLETF rats for each category.

in the management of type 2 diabetes. In this study, we investigated the suitability of maple syrup by comparing the changes in PG levels after the oral administration of sucrose or maple syrup using OLETF rats as a model for type 2 diabetes.

In work such as this to study accurately the effects of the oral administration of sucrose or maple syrup on PG levels in diabetes mellitus, the selection of the experimental animal is very important. Animal models used to investigate the diabetes mellitus include streptozotocin-induced
Table $3 A U C_{0-180 \min }$ for PG in 30- and 60-week-old LETO and OLETF rats following the oral administration of sucrose or maple syrup.

\begin{tabular}{lcc}
\hline & & $A U C_{0-180 \min }(\mathrm{mg} \cdot \mathrm{min} / \mathrm{dl})$ \\
\hline LETO & Sucrose & $7830 \pm 510$ \\
(30 week) & Maple & $5975 \pm 508^{*}$ \\
OLETF & Sucrose & $13200 \pm 1296$ \\
(30 week) & Maple & $10355 \pm 686^{*}$ \\
LETO & Sucrose & $7598 \pm 320$ \\
(60 week) & Maple & $5974 \pm 878^{*}$ \\
OLETF & Sucrose & $12525 \pm 816$ \\
(60 week) & Maple & $9062 \pm 336^{*}$ \\
\hline
\end{tabular}

The data are presented as means \pm S.E. of 3 independent rats. $* p<0.05$ vs. sucrose for each category.

diabetic rats as an animal model for insulin-dependent diabetes mellitus ${ }^{21)}$. Although hyperglycemia is present in streptozotocin-induced diabetic rats, the general pathophysiology of the model differs from that of non-insulin-dependent diabetes mellitus in humans. On the other hand, the general pathophysiology of GK (Goto-Kakizaki) rats, which are used as a model for type 2 diabetes mellitus, can be used for similar purposes ${ }^{22}$. However, GK rats develop type 2 diabetes mellitus in the absence of metabolic syndrome. The OLETF rat is an established model of human type 2 diabetes mellitus ${ }^{20)}$ in which nearly $100 \%$ of males develop a diabetic syndrome by 25 weeks of age. The food intake and water consumption in OLETF rats are higher, and the body weight and TG levels of OLETF rats are significantly increased than that in LETO rats. This enhancement of TG levels causes insulin resistance. Thereby, hyperglycemia and hyperinsulinemia are exhibited in the early phase of the disease as a result of islet cell hyperplasia and peripheral IR at 30 weeks of age ${ }^{23-25)}$. With increasing age, the rats eventually develop hypoinsulinemia as a result of $\beta$-cell islet deterioration caused by $\operatorname{ROS}^{25)}$; the plasma insulin levels in OLETF rats over 60 weeks of age are lower than those in LETO rats of the corresponding age used as normal controls ${ }^{26,27)}$. We have also reported that the oral administration of a powerful antioxidant drug (disulfiram) to OLETF rats prevents the increase in glucose levels $^{28)}$, and that OLETF rats can be used as a model of diabetic complications, such as diabetic keratopathy and cat$\operatorname{aracts}^{29-31)}$. These changes in the biological characteristics of OLETF rats correspond to those of human type 2 diabetes mellitus. Therefore, OLETF rats may provide a useful model of type 2 diabetes for studying the changes in PG and insulin levels following the administration of maple syrup.

The body weights of 30-week-old OLETF rats were approximately 1.3 -fold higher than those of 30 -week-old 

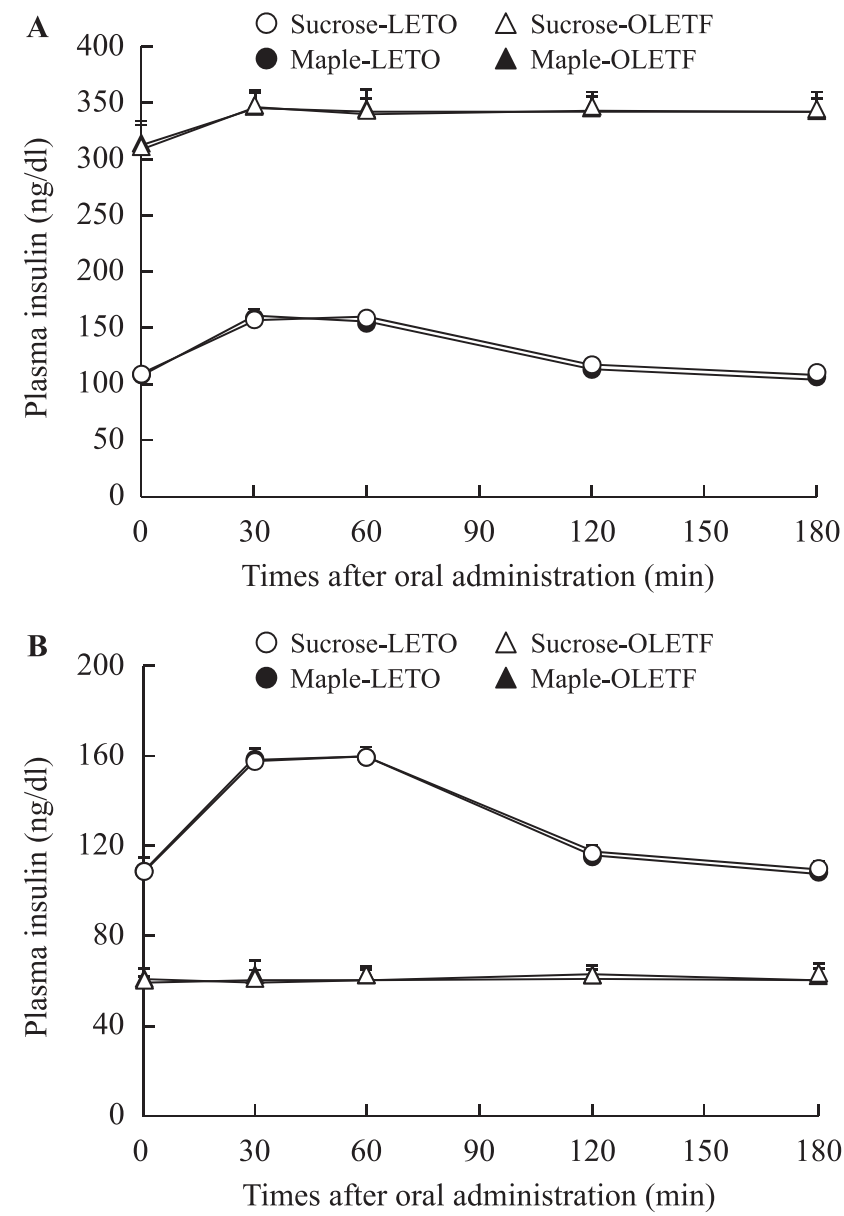

Fig. 2 Changes in plasma insulin levels in 30 (A)and $60(\mathrm{~B})$-week-old LETO and OLETF rats following the oral administration of sucrose or maple syrup. The insulin level was determined using the ELISA method. Open circles, sucroseadministered LETO rats; closed circles, maple syrup-administered LETO rats; open triangles, sucrose-administered OLETF rats; closed triangles, maple syrup-administered OLETF rats. The data are presented as means \pm S.E. of 3 independent rats.

LETO rats (Table 2). The PG, TG and Total-cho levels in OLETF rats increased with age, and reached plateaus within 30 weeks. The plasma insulin levels in 30 -week-old OLETF rats were also higher than those in the same age LETO rats (Table 2). These results indicate that 30-weekold OLETF rats had developed diabetes mellitus via IR. Consistent with the results in 30-week-old OLETF rats, the body weights and plasma insulin levels of 60 -week-old OLETF rats were lower than those of 60-week-old LETO rats (Table 2). Hirashima et al..$^{26)}$ reported atrophy or the disappearance of $\beta$ cells in OLETF rats older than 60 weeks of age, and found that plasma insulin levels were lower
Table $4 A U C_{0-180 \min }$ for insulin in 30- and 60-weekold LETO and OLETF rats following the oral administration of sucrose or maple syrup.

\begin{tabular}{lcc}
\hline & & $A U C_{0-180 \min }(\mathrm{mg} \cdot \mathrm{min} / \mathrm{dl})$ \\
\hline LETO & Sucrose & $3247 \pm 435$ \\
(30 week) & Maple & $2919 \pm 823$ \\
OLETF & Sucrose & $3465 \pm 267$ \\
(30 week) & Maple & $3408 \pm 1097$ \\
LETO & Sucrose & $3465 \pm 736$ \\
(60 week) & Maple & $3177 \pm 642$ \\
OLETF & Sucrose & $281 \pm 163$ \\
(60 week) & Maple & $169 \pm 40$ \\
\hline
\end{tabular}

The data are presented as means \pm S.E. of 3 independent rats.

than in LETO rats $^{27)}$. These findings suggest that the type 2 diabetes mellitus in 60-week-old OLETF rats has reached a fairly advanced stage, and that the changes may be due to the deterioration of islet beta cells with disease progression.

In this study, we compared changes of PG levels in OLETF rats following the oral administration of sucrose or maple syrup, and demonstrated the relationships between PG and insulin levels using 30- and 60-week-old OLETF rats with hyperglycemia and hyperinsulinemia to investigate whether maple syrup is suitable as a sweetener in the management of type 2 diabetes for medium (30 weeks of age) and critical ( 60 weeks of age) degree. The insulin levels in OLETF rats at 30 weeks of age increased following the oral administration of either sucrose or maple syrup, and after 3 hours had not yet decreased to the pre-prandial levels (Fig. 2). On the other hand, the insulin levels in OLETF rats at 60 weeks of age did not increase in response to the oral administration of sucrose or maple syrup. No significant differences were observed in the insulin levels between sucrose- and maple syrup-administered LETO or OLETF rats at 30 or 60 weeks of age (Table 4). The results show that insulin is not functioning in 60-week-old OLETF rats. On the other hand, the enhancements of PG in 30and 60-week-old OLETF rats administered maple syrup were lower than when the same rats were administered sucrose, and this effect was not due to insulin enhancement, since the $A U C_{0-180 \text { min }}$ values for PG in OLETF rats administered maple syrup were significantly lower than those for sucrose-administered OLETF rats at 60 weeks of age which insulin was not functioning (Fig. 1). In addition, the $A U C_{0-180}$ min values for PG in LETO rats administered maple syrup were also significantly lower than those for sucrose-administered LETO rats without IR (Table 3 ). It is known that sucrose, which is disaccharide, decomposes to glucose(monosaccharide)through the action of $\alpha$-glucosidase in epithelial cells of the small intestine, and 
Apostolidis et $a l .{ }^{19)}$ reported that maple syrup has an $\alpha$-glucosidase inhibitory activity in in vitro. Taken together, the data suggest that the absorption ratio of glucose from maple syrup may be lower than from sucrose in the small intestine, thus preventing the enhancement of $P G$ in maple syrup-administered OLETF rats.

Further studies are needed to identify the compounds responsible for the observed low glucose absorption following the oral administration of maple syrup. In addition, it is important to clarify the preventive effect of maple syrup on the development of type 2 diabetes mellitus. We will next investigate the effect of maple syrup supplementation on body weight and blood test values for diabetes mellitus (PG, TG, Total-cho and insulin) in OLETF rats during the development of diabetes. In preparation for future work, we have started to identify the compounds related to the suppression of glucose absorption from maple syrup, and found some unknown peaks that have not been reported, on electropherograms obtained from our series of our study by capillary electrophoresis. We will investigate the relationships of those unknown peaks and low glycemic index of maple syrup. These results support the previous in vitro study ${ }^{19)}$, and contribute to our understanding of the observed health benefits and biological activities of maple syrup.

\section{CONCLUSION}

The present study demonstrates that the increase in PG following the oral administration of maple syrup is lower than in the case of sucrose administration in LETO and OLETF rats, and that the phenomenon may be caused by the low absorption ratio of glucose via maple syrup. These findings provide significant information concerning the potential for the use of maple syrup as a sweetener in the management of type 2 diabetes, and indicate that maple syrup with its lower glycemic index may be useful in the prevention of type 2 diabetes.

\section{References}

1) Haffner, S. M.; Valdez, R. A.; Hazuda, H. P.; Mitchell, B. D.; Morales, P. A.; Stern, M. P. Prospective analysis of the IR syndrome (syndrome X). Diabetes 41, 715-722 (1992).

2) Isomaa, B.; Almgren, P.; Tuomi, T.; Forsen, B.; Lahti, K.; Nissen, M.; Taskinen, M.R.; Groop, L. Cardiovascular morbidity and mortality associated with the metabolic syndrome. Diabetes Care 24, 683-689 (2001).

3) Porte, D. Banting lecture 1990. Beta-cells in type II diabetes mellitus. Diabetes 40, 166-180 (1991).

4) DeFronzo, R. A.; Bonadonna, R. C.; Ferrannini, E.
Pathogenesis of NIDDM. A balanced overview. Diabetes Care 15, 318-368(1992).

5) Yki, J. H. Glucose toxicity. Endocrine. Rev. 13, 415431 (1992).

6) Vinik, A.; Pittenger, G.; Rafaeloff, R.; Rosenberg, L.; Duguid, W. Determinants of pancreatic islet cell mass: a balance between neogenesis and senescence/apoptosis. Diabetes Rev. 4, 235-263(1996).

7) Matsuoka, T.; Kajimoto, Y.; Watada, H.; Kaneto, H.; Kishimoto, M.; Umayahara, Y.; Fujitani, Y.; Kamada, T.; Kawamori, R.; Yamasaki, Y. Glycation-dependent, reactive oxygen species-mediated suppression of the insulin gene promoter activity in HIT cells. J. Clin. Invest. 99, 144-150 (1997).

8) Kaneto, H.; Fujii, J.; Myint, T.; Miyazawa, N.; Islam, K.N.; Kawasaki, Y.; Suzuki, K.; Nakamura, M.; Tatsumi, H.; Yamasaki, Y.; Taniguchi, N. Reducing sugars trigger oxidative modification and apoptosis in pancreatic beta-cells by provoking oxidative stress through the glycation reaction. Biochem. J. 320, 855-863(1996).

9) Tiedge, M.; Lortz, S.; Drinkgern, J.; Lenzen, S. Relation between antioxidant enzyme gene expression and antioxidative defense status of Insulin-producing cells. Diabetes. 46, 1733-1742(1997).

10) Ball, D. W. The chemical composition of maple syrup. Journal of Chemical Education 84, 1647-1650 (2007).

11) Davison, R. M.; Young, H. Abscisic acid content of xylem sap. Planta (Berl.) 109, 95-98 (1973).

12) Perkins, T. D.; van den Berg, A. K. Maple syrupproduction, composition, chemistry, and sensory characteristics. Adv. Food Nutr. Res. 56, 101-143(2009).

13) Taga, A.; Sato, A.; Suzuki, K.; Takeda, M.; Kodama, S. Simple determination of a strongly aromatic compound, sotolon, by capillary electrophoresis. J. Oleo Sci. 61, 45-48(2012).

14) Taga, A.; Kodama, S. Analysis of reducing carbohydrates and fructosyl saccharides in maple syrup and maple sugar by CE. Chromatographia. 75, 1009-1016 (2012).

15) Li, L.; Seeram, N. P. Maple syrup phytochemicals include lignans, coumarins, a stilbene, and other previously unreported antioxidant phenolic compounds. Agric Food Chem. 58, 11673-11679 (2010).

16) Li, L.; Seeram, N. P. Further investigation into maple syrup yields 3 new lignans, a new phenylpropanoid, and 26 other phytochemicals. Agric Food Chem. 59, 7708-7716 (2011).

17) Li, L.; Seeram, N. P. Quebecol, a novel phenolic compound isolated from Canadian maple syrup. J. Funct. Foods. 2, 125-128(2011).

18) Legault, J.; Girard-Lalancette, K.; Grenon, C.; Dussault, C.; Pichette, A., Antioxidant activity, inhibition of nitric oxide overproduction, and in vitro antip- 
roliferative effect of maple sap and syrup from Acer saccharum. J. Med. Food 13, 460-468(2010).

19) Apostolidis; E., Li; L., Lee; C., Seeram NP. In vitro evaluation of phenolic-enriched maple syrup extracts for inhibition of carbohydrate hydrolyzing enzymes relevant to type 2 diabetes management. J. Funct. Foods. 3, 100-106 (2011).

20) Kawano, K.; Hirashima, T.; Mori, S.; Saitoh, Y.; Kurosumi, M.; Natori, T. Spontaneous long-term hyperglycemic rat with diabetic complications. Otsuka LongEvans Tokushima Fatty (OLETF) strain. Diabetes. 41, 1422-1428(1992).

21) Prager; R., Abramovici; A., Liban; E., Laron; Z. Histopathological changes in the placenta of streptozotocin induced diabetic rats. Diabetologia. 10, 89-91 (1974).

22) Wakuta; M., Morishige; N., Chikama; T., Seki; K., Nagano; T., Nishida; T. Invest. Ophthalmol. Vis. Sci. 48, 590-596 (2007).

23) Ishida, K.; Mizuno, A.; Min, Z.; Sano, T.; Shima, K. Which is the primary etiologic event in Otsuka LongEvans Tokushima Fatty rats, a model of spontaneous non-insulin-dependent diabetes mellitus, insulin resistance, or impaired insulin secretion? Metabolism. 44, 940-945 (1995).

24) Sato, T.; Asahi, Y.; Toide, K.; Nakayama, N. Insulin resistance in skeletal muscle of the male Otsuka LongEvans Tokushima Fatty rat, a new model of NIDDM. Diabetologia. 38, 1033-1041(1995).

25) Yabuki, A.; Tahara, T.; Taniguchi, K.; Matsumoto, M.; Suzuki, S. Neuronal nitric oxide synthase and cyclooxygenase-2 in diabetic nephropathy of type 2 diabetic
OLETF rats. Exp. Anim. 55, 17-25 (2006).

26) Hirashima, T.; Man, Z. W.; Mori, S.; Kawano, K. OLETF rat. Diabetes Frontier 9, 477-480 (1998).

27) Minami, A.; Ishimura, N.; Sakamoto, S.; Takishita, E.; Mawatari, K.; Okada, K.; Nakaya, Y. Effect of eicosapentaenoic acid ethyl ester v. oleic acid-rich safflower oil on insulin resistance in type 2 diabetic model rats with hypertriacylglycerolaemia. Br. J. Nutr. 87, 157162 (2002).

28) Nagai, N.; Murao, T.; Okamoto, N.; Ito, Y. Disulfiram reduces elevated blood glucose levels in Otsuka LongEvans Tokushima Fatty (OLETF) rats, a model of type 2 diabetes. J. Oleo Sci. 58, 485-490 (2009).

29) Nagai, N.; Murao, T.; Ito, Y.; Okamoto, N.; Sasaki, M. Enhancing effects of sericin on corneal wound healing in Otsuka Long-Evans Tokushima fatty rats as a model of human type 2 diabetes. Biol. Pharm. Bull. 32, 1594-1599 (2009).

30) Nagai, N.; Murao, T.; Okamoto, N.; Ito, Y. Kinetic analysis of the rate of corneal wound healing in Otsuka long-evans Tokushima Fatty rats, a model of type 2 diabetes mellitus. J. Oleo Sci. 59, 441-449 (2010).

31) Nagai, N.; Murao, T.; Ito, Y.; Okamoto, N.; Okamura, H. Involvement of interleukin 18 in lens opacification of Otsuka Long-Evans Tokushima Fatty rats, a model of human type 2 diabetes. Curr. Eye Res. 36, 497-506 (2011). 